\title{
Differential Inequalities on Complete Riemannian Manifolds and Applications
}

\author{
Leon Karp ${ }^{\star}$ \\ Department of Mathematics, University of Michigan, Ann Arbor, MI 48109, USA
}

\section{Introduction}

This paper treats various aspects of the asymptotic behavior of solutions of certain elliptic equations of geometric interest on complete Riemannian manifolds.

Sharp results relating the rate of volume growth of a complete Riemannian manifold and the growth of its harmonic and subharmonic functions can be found in [22] together with references to related results. In Sect. 2 of this paper we consider solutions of the more restrictive inequality $\Delta u \geqq \varepsilon>0$ (where $\Delta=$ div $\circ$ grad) and show that these must be unbounded if $M^{n}$ has even quadratically exponential volume growth, and that $u$ must have faster than linear growth if $M^{n}$ has sub-exponential volume growth (as does the function $u=x^{2}$ on $\mathbb{R}^{1}$ for which $\frac{d^{2}}{d x^{2}} u=2$ ). These results (which are sharp) lead, in Sect. 3 to an inequality for the exterior radius of certain complete Riemannian manifolds immersed with bounded mean curvature vector in Euclidean space. Our results in this direction generalize previous results of Aminov [1], Hasanis and Koutroufiotis [14], and Jorge and Xavier [20]. In particular, it is shown that a complete Riemannian manifold with exponential (or even quadratically exponential) volume growth cannot be minimally immersed in a bounded set in Euclidean space, while it is known ([18], cf. [34, p. 690]) that such immersions exist if the volume growth restrictions are removed.

The last section of the paper connects the results of Sect. 2 with Brownian motion on complete minimal submanifolds of $\mathbb{R}^{N}$, and it is shown that Brownian motion is non-explosive on a complete, properly imbedded minimal submanifold of $\mathbb{R}^{N}$.

Weaker versions of some of the results of this paper were stated without proof in [23].

* Research supported by NSF grants MCS 79-019147 and 81-02051 


\section{The Main Analytic Theorems}

In this section and throughout the paper we let $\varrho(\cdot)$ denote the intrinsic Riemannian distance function on the Riemannian manifold $\left(M^{n}, d s^{2}\right)$; the distance is measured from a fixed point $x_{0} \in M$ which plays no role in what follows. We denote by $B\left(x_{0} ; r\right)$ the geodesic ball of radius $r$ and center $x_{0}$, and we often write $B(r)$ when the specific choice of $x_{0}$ is irrelevant.

In what follows we will use two quantitative measures of the rate of volume growth at infinity the Riemannian manifold $\left(M^{n}, d s^{2}\right)$. We define

$$
\gamma_{1}=\limsup _{r \rightarrow \infty} \frac{1}{r} \log \operatorname{vol} B(r)
$$

and

$$
\gamma_{2}=\limsup _{r \rightarrow \infty} \frac{1}{r^{2}} \log \operatorname{vol} B(r) .
$$

It is well-known that the rate of volume growth is connected with lower estimates for the Ricci tensor. Indeed, one has the following standard estimates:

Proposition 2.1 (cf. [4, p. 256]). If the Ricci curvature of $M^{n}$ satisfies

$$
\text { Ricci } \geqq-(n-1) \alpha^{2}
$$

in $B(r)$ then

$$
\operatorname{vol} B(r) \leqq c_{n} \int_{0}^{r} \frac{(\sinh \alpha t)^{n-1}}{\alpha^{n-1}} d t
$$

As an immediate consequence we have

Corollary 2.1.1. If $\left(M^{n}, d s^{2}\right)$ is complete and the Ricci tensor satisfies

$$
\text { Ricci } \geqq-(n-1) \alpha^{2}
$$

then $\gamma_{1} \leqq(n-1) \alpha<\infty$.

If we let $\alpha$ in (2.1) be dependent on $r$ then we may derive

Corollary 2.1.2. If $\left(M^{n}, d s^{2}\right)$ is complete and the Ricci tensor satisfies

$$
\operatorname{Ricci}_{x} \geqq-c\left(1+\varrho(x)^{2}\right)
$$

for some constant $c>0$ and all points $x$ then $\gamma_{2}<\infty$.

It is worth remarking that this last estimate is essentially sharp. For example, the metric $d s^{2}=d r^{2}+G(r)^{2} d \theta^{2}$ on $\mathbf{R}^{2}$ [where $G(r)>0$ if $r>0, G(0+)=0$, and $\left.G^{\prime}(0+)=1\right]$ has $\gamma_{2}=+\infty$ if $G(r)=e^{r^{2+s}}$ for $r \geqq 1$, and in this case the curvature $K_{x}=-G^{\prime \prime} / G \sim-\varrho(x)^{2+2 \varepsilon}$. On the other hand, a consideration of metrics with $G(r)=e^{-f(r)}, f(r) \rightarrow \infty$, and $f^{\prime \prime}-f^{\prime 2} \rightarrow-\infty$ shows that we can have $\operatorname{vol}(M)<\infty$ and still have curvatures tending to $-\infty$ with arbitrary speed as $\varrho(x) \rightarrow \infty$.

We now turn to the main results of the paper: 
Theorem 2.2. Let $\left(M^{n}, d s^{2}\right)$ be a complete Riemannian manifold with volume-growth of exponential type (i.e., $\left.\gamma_{1}<\infty\right)$ ). If $u: M^{n} \rightarrow \mathbb{R}^{1}$ satisfies

$$
\limsup _{e \rightarrow \infty}\left(\frac{u(x)}{\varrho(x)}\right)<a
$$

then

$$
\inf _{M}(\Delta u)<\gamma_{1} a .
$$

A related result is contained in

Theorem 2.3. Let $\left(M^{n}, d s^{2}\right)$ be a complete Riemannian manifold with $\gamma_{2}<\infty$. If $u: M^{n} \rightarrow \mathbb{R}^{1}$ satisfies supu $<\infty$ then

$$
\inf \Delta u \leqq 0 .
$$

We postpone for a moment the proofs of Theorems 2.2 and 2.3 and turn to some

Examples. 1) Let $\left(M^{n}, d s^{2}\right)$ be a simply-connected space of constant negative sectional curvature $\equiv-c^{2}$. It is known (cf. [13, Chap. 2]) that on such a manifold $\Delta \varrho \geqq(n-1) c$ and $\gamma_{1}=(n-1) c$. If we now take $u=\varrho$ it follows that the statement of Theorem 2.3 is sharp.

2) Consider the metric $d s^{2}=d r^{2}+G(r)^{2} d \theta^{2}$ on $\mathbb{R}^{2}$. One checks easily that, for a radial function $u(r)$,

$$
\Delta u=\frac{1}{G}\left(G u_{r}\right)_{r}
$$

It follows that the function $u(r)=\int_{0}^{r} \frac{d s}{G(s)} \int_{0}^{s} G(t) d t$ satisfies $\Delta u \equiv 1$ and

$$
\sup u(r)=\int_{0}^{\infty} \frac{d s}{G(s)} \int_{0}^{s} G(t) d t .
$$

Note that this improper integral converges if we take $G(r)=r^{1+\varepsilon} e^{r^{2+\varepsilon}}$ for $r \geqq 1$, and in this case $\log \operatorname{vol} B(r) \sim r^{2+\varepsilon}$ as $r \rightarrow \infty$, and $\gamma_{2}$ just barely fails to be finite. Theorem 2.3 is thus quite sharp.

We now give the

Proof of Theorem 2.2. We first note that given $0<\theta<1$ there exists a positive constant $C>0$ and a family $\{\phi(\cdot ; r)\}, r \in \mathbb{R}^{+}$, of smooth functions with compact support such that $\phi(x ; r) \equiv 1$ for $x \in B(\theta r), \phi(x ; r) \equiv 0$ for $x \in M^{n}-B(r)$, and $\|\nabla \phi(x ; r)\| \leqq C$ for all $x \in M^{n}$ and $r \in \mathbb{R}^{+}$. In fact, it is shown in [12] that given $\varepsilon>0$ there exists a $C^{\infty}$ approximation $\psi$ to the distance function $\varrho$ such that $\sup |\psi-\varrho| \leqq \varepsilon$ and $\|\nabla \psi\| \leqq 1+\varepsilon$. A family with the desired properties may be defined via $\phi=\mu(\psi)$ after an appropriate choice of $\mu \in C_{0}^{\infty}\left(\mathbf{R}^{1}\right)$. (For a similar construction see [2] or [32].)

With $\lambda>0$ (to be chosen later) and $\varrho=\varrho(x)$, we have

$$
\operatorname{div}[\exp (\lambda u-\gamma \varrho) \cdot \nabla u]=\exp (\lambda u-\gamma \varrho)\left[\lambda\|\nabla u\|^{2}-\gamma\langle\nabla \varrho, \nabla u\rangle+\Delta u\right] .
$$


Now if inf $\Delta u=b>0$ we have

$$
\lambda\|\nabla u\|^{2}-\gamma\|\nabla u\|+\Delta u \geqq\left(\lambda-\frac{\gamma^{2}}{4 \beta}\right)\|\nabla u\|^{2}
$$

for every $\beta<b$. It follows that with $\lambda-\frac{\gamma^{2}}{4 \beta}=\Lambda(\lambda, \gamma, \beta)=\Lambda>0$

$$
\begin{aligned}
A & \int_{B(r)} \phi^{2}(x ; r) \exp (\lambda u-\gamma \varrho)\|\nabla u\|^{2} \\
\leqq & 2 \int_{B(r)} \phi(x ; r) \cdot \exp (\lambda u-\gamma \varrho)|\langle\nabla \phi, \nabla u\rangle| \\
\leqq & 2\left(\int_{B(r)} \phi^{2} \exp (\lambda u-\gamma \varrho)\|\nabla u\|^{2}\right)^{1 / 2} \\
& \cdot\left(\int_{B(r)}\|\nabla \phi\|^{2} \exp (\lambda u-\gamma \varrho)\right)^{1 / 2},
\end{aligned}
$$

since $\int_{B(2 r)} \operatorname{div}\left(\phi^{2} X\right)=0$ for any vector field $X$. Now $\nabla \phi \equiv 0$ except in the annular region $B(r)-B(\theta r)$. Thus if $\gamma=\gamma_{1}+\eta+\delta$

$$
\int_{B(r)}\|\nabla \phi\|^{2} \exp (\lambda u-\gamma \varrho) \leqq c^{2} \sup _{B(r)-B(\theta r)}[\exp (\lambda u-\eta \varrho)] \int_{B(r)-B(\theta r)} e^{-\left(\gamma_{1}+\delta\right) e}
$$

It follows from the definition of $\gamma_{1}$ that

$$
\int_{M} e^{-\left(\gamma_{1}+\delta\right) e}<\infty
$$

in fact,

$$
\int_{M} e^{-\left(\gamma_{1}+\delta\right) e}=\sum_{k=1}^{\infty} \int_{B(k)-B(k-1)} e^{-\left(\gamma_{1}+\delta\right) e} \leqq e^{\gamma_{1}+\delta} \sum_{k=1}^{\infty} e^{-\left(\gamma_{1}+\delta\right) k} \operatorname{vol} B(k)
$$

which converges. Consequently, if eventually $\lambda u-\eta \varrho \leqq 0$ as $\varrho \rightarrow \infty$, it follows from (2.1), (2.2), and the fact that

$$
\int_{B(r)-B(\theta r)} e^{\left(-y_{1}+\delta\right) e} \rightarrow 0
$$

as $r \rightarrow \infty$, that $\|\nabla u\| \equiv 0$. This contradicts the assumption that $\inf _{M} \Delta u>0$. To complete the proof we note that if $\limsup _{r \rightarrow \infty}\left(\frac{u}{\varrho}\right)<a$ then $\frac{\eta}{a} u-\eta \varrho<0$, eventually. If $\frac{\left(\gamma_{1}+\eta\right)^{2}}{\eta}<\frac{4 b}{a}$ then we can choose $\lambda$ with $\frac{\gamma^{2}}{4 \beta}<\lambda<\frac{\eta}{a}$ for some $\delta=\gamma-\eta-\gamma_{1}>0$ and $0<\beta<b$, and then $\lambda u-u \varrho \leqq 0$ for $\varrho$ large. Set $F(\eta)=\left(\frac{\gamma_{1}}{\sqrt{\eta}}+\sqrt{\eta}\right)^{2} . F$ achieves a minimum value of $4 \gamma_{1}$ with the choice $\eta=\gamma_{1}$. It follows that if $\frac{b}{a}>\gamma_{1}$, we are led to a contradiction.

We now turn to the indirect

Proof of Theorem 2.3. Without loss of generality we may assume that $u<0$. If $\Delta u \geqq b>0$ on $M^{n}$ then $v=\frac{1}{b} u$ satisfies $\Delta v \geqq 1$ and $w=e^{v}$ satisfies $0<w<1$, and 
$\Delta w \geqq e^{v} \Delta v \geqq w$. Consequently, the function $\phi(x, t)==_{\mathrm{def}}\left(e^{t}-1\right) w-t$ satisfies $\left(\Delta-\frac{\partial}{\partial t}\right) \phi=\left(e^{t}-1\right) \Delta w-e^{t} w+1 \geqq\left(e^{t}-1\right) w-e^{t} w+1 \geqq 0$ on $M x[0, \infty)$.

Let $\psi_{n}$ be the solution of the heat equation $\left(\Delta-\frac{\partial}{\partial t}\right) \psi_{n}=0$ on $\Omega_{n}=K_{n} \times[0, T]$ with $\psi_{n}=\phi$ on $\partial_{0} \Omega_{n}={ }_{\text {def }} K_{n} \times\{t=0\} \cup \partial K_{n} \times[0, T]$. Here $\left\{K_{n}\right\}$ is a smoothly bounded exhaustion of $M^{n}$, and $T>0$ will be chosen later. Since $\phi$ is uniformly bounded on $M x[0, T]$ it follows from the maximum principle that the functions $\left\{\psi_{n}\right\}$ are also uniformly bounded on $K \times[0, T]$ if $K \subset \subset M$, and $n \gg 1$. By standard interior estimates for parabolic equations [11] the functions $\left\{\psi_{n}\right\}$ converge to a global solution $\psi$ of the heat equation on $M \times[0, T]$ and $\psi(x, 0)=0$ since $\phi=0$ when $t=0$. We claim that $\psi \neq 0$. Indeed,

$$
\left(\Delta-\frac{\partial}{\partial \mathrm{t}}\right)\left[\psi_{n}-\phi\right] \leqq 0,
$$

in $\Omega_{n}$ and $\psi_{n}=\phi$ on $\partial_{0} \Omega_{n}$. It follows that $\psi_{n} \geqq \phi$ in $\Omega_{n}$. Now consider the difference $\psi_{n+1}-\psi_{n}$ in $\Omega_{n}$. We have $\left(A-\frac{\partial}{\partial t}\right)\left(\psi_{n+1}-\psi_{n}\right)=0$ and $\psi_{n+1}-\psi_{n}=\psi_{n+1}-\phi \geqq 0$ on $\partial_{0} \Omega_{n}$. It follows that the sequence $\left\{\psi_{n}\right\}$ is increasing, and $\psi \geqq \psi_{n}$ for all $n$. Now $\psi_{n}(x, t) \geqq\left(e^{t}-1\right) w-t>0$ for $t$ large enough and $x$ fixed. Therefore, if we choose $T$ large enough, $\psi(x, t)>0$ for some $x \in M$ and some $t<T$. It follows that $\psi$ is a solution of the heat equation that is uniformly bounded on $M \times[0, T]$, initially equal to zero and not identically zero. This, however, contradicts a recent result of Karp and Li [24] which asserts that no such function can exist if $\gamma_{2}<\infty$. This completes the proof.

Remarks. (a) Theorem 2.3 generalizes a result asserted without proof (and in a different context in [15]).

(b) Theorems 2.2 and 2.3 may be considered as "weak generalized maximum principles" on noncompact manifolds. Note that if there exists a point $p \in M^{n}$ for which $u(p) \geqq u(x), \forall x \in M^{n}$, then $\Delta u(p) \leqq 0$. Such a point certainly exists, and has the additional property that $\nabla u(p)=0$, if $M^{n}$ is compact. Generalizing earlier work of Omori [27], it was shown by Yau [31] (see also $[7,14]$ ) that if the Ricci curvature of $\left(M^{n}, d s^{2}\right)$ is bounded from below and $\left(M^{n}, d s^{2}\right)$ is complete then a generalized maximum principle is valid: If $u: M^{n} \rightarrow \mathbb{R}^{1}$ satisfies sup $u<\infty$ then given $\varepsilon>0, \exists p_{\varepsilon} \in M^{n}$ with $\Delta u\left(p_{\varepsilon}\right) \leqq \varepsilon,\left\|\nabla u\left(p_{\varepsilon}\right)\right\| \leqq \varepsilon$, and $u\left(p_{\varepsilon}\right)>\sup u-\varepsilon$. This result is extremely useful (see [33], for instance) and it is reasonable to ask whether or not Theorem 2.2 (resp. 2.3) can be strengthened to give this conclusion under the weaker hypothesis that $\gamma_{1}<\infty$ and $\limsup _{a \rightarrow \infty}(u(x) / \varrho(x)) \leqq 0$ (resp., $\gamma_{2}<\infty$ and $\sup u<\infty)$. It may be noted that for any complete manifold $\left(M^{n}, d s^{2}\right)$, if $u: M^{n} \rightarrow \mathbb{R}^{1}$ satisfies $\sup u<\infty$ then given $\varepsilon>0 \exists p_{\varepsilon}$ with

$$
u\left(p_{\varepsilon}\right)>\sup u-\varepsilon \text { and }\left\|\nabla u\left(p_{\varepsilon}\right)\right\| \leqq \sqrt{\varepsilon} .
$$

This follows from very general metric space results [17].

We conclude this section with some immediate consequences of the theorems and Proposition 2.1. 
Corollary 2.2.1. Let $\left(M^{n}, d s^{2}\right)$ be complete with Ricci curvature bounded below by $-(n-1) c^{2}$. If $u: M^{n} \rightarrow \mathbb{R}^{1}$ satisfies $\limsup _{\boldsymbol{Q} \rightarrow \infty}(u(x) / \varrho(x))<a\left(\right.$ resp., $\left.\sup _{\boldsymbol{N}}|\nabla u|<\infty\right)$ then $\inf (\Delta u)<(n-1) c a($ resp., $\inf \Delta u \leqq(n-1) c \cdot \sup |\nabla u|)$. If u has sublinear growth (i.e., $a=0$ ) then $\inf (\Delta u) \leqq 0$.

Corollary 2.3.1. Let $\left(M^{n}, d s^{2}\right)$ be complete with Ricci curvature satisfying $\operatorname{Ricci}_{x} \geqq$ $-c\left(1+\varrho^{2}(x)\right)$ for some $c>0$. If $u: M^{n} \rightarrow \mathbb{R}^{1}$ and $\sup u<\infty$ then $\inf (\Delta u) \leqq 0$.

Corollary 2.2.2. Let $\left(M^{n}, d s^{2}\right)$ be complete and have subexponential volume growth (which occurs, for instance, if Ricci $\geqq 0$ ). If $u: M^{n} \rightarrow \mathbb{R}^{1}$ and $\sup |\nabla u|<\infty$ then $\inf (\Delta u) \leqq 0$.

Remark. A result somewhat weaker than Theorems 2.2 and 2.3 was announced without proof in the author's paper [23].

\section{Applications: Isometric Immersions and Minimal Submanifolds}

In this section we consider various applications of the results of Sect. 2. We begin with an estimate for the exterior radius of a complete manifold immersed in Euclidean space (or any simply-connected space of nonpositive curvature). Recall that according to a result of Nash [26] any noncompact n-dimensional Riemannian manifold $\left(M^{n}, d s^{2}\right)$ can be isometrically imbedded in a ball of preassigned radius $\varepsilon>0$ in $\mathbb{R}^{n+k}$ if the codimension $k$ is large enough. Of course, such an imbedding would have to twist a great deal and this twisting is controlled by the magnitude of mean curvature vector $H\left(=\frac{1}{n}\right.$ trace of the second fundamental form) of the immersion.

Theorem 3.1. Let $\left(M^{n}, d s^{2}\right)$ be a complete Riemannian manifold with volume growth satisfying $\gamma_{2}<\infty$. If $\left(M^{n}, d s^{2}\right)$ is isometrically immersed in a simply-connected complete Riemannian manifold $\bar{M}^{n+k}, k>0$ of nonpositive curvature and $H_{0}=\sup _{M}\|H\|<\infty$, then the radius $R$ of the smallest geodesic ball in $\bar{M}^{n+k}$ that contains $M^{n}$ satisfies $R \geqq \frac{1}{H_{0}}$.

Remark. Clearly, $R=\frac{1}{H_{0}}$ if $M^{n}$ is a hypersphere of radius $R$ in $\mathbb{R}^{n+1}$.

Corollary 3.1.1. Let $\left(M^{2}, d s^{2}\right)$ be a complete Riemannian manifold with scalar curvature $S$ that satisfies $S(x) \geqq-C\left(1+Q(x)^{2}\right)$ for some $C>0$. If $\left(M^{n}, d s^{2}\right)$ is is isometrically immersed in geodesic ball of radius $R$ in a simply-connected space of nonpositive curvature then $R \geqq \frac{1}{H_{0}}, H_{0}=\sup _{M}\|H\|$.

A particular case deserves special mention:

Corollary 3.1.2. If $\left(M^{n}, d s^{2}\right)$ is a complete Riemannian manifold with scalar curvature $S$ that satisfies $S(x) \geqq-C\left(1+Q(x)^{2}\right)$ for some $C>0$, or if $\gamma_{2}<\infty$ (e.g., if 
$\operatorname{vol} M<\infty)$, then $\left(M^{n}, d s^{2}\right)$ cannot be isometrically minimally immersed in a bounded set in a simply-connected space of nonpositive curvature.

To prove these results we need

Lemma 3.2. Suppose that $M^{n}$ is isometrically immersed in $\bar{M}^{n+k}$. If $f: \bar{M} \rightarrow \mathbb{R}^{1}$ then the Laplacian of the restriction of $f$ to $M$ is given by

$$
\Delta_{M} f=\operatorname{tr}_{M}\left(\bar{V}^{2} f\right)+n\left\langle H, \operatorname{grad}_{\bar{M}} f\right\rangle_{\bar{M}} .
$$

Here $\bar{\nabla}$ is the Riemannian connection on $\bar{M}$ and $H$ is the mean curvature vector of the immersion $=\frac{1}{n}$ (trace of the second fundamental form).

This formula is well-known, but since it is difficult to give an explicit reference we give a short proof for completeness.

Proof. Since $A_{M}=\operatorname{div}_{M} \circ \operatorname{grad}_{M}$ and $\operatorname{div}_{M} X=\Sigma\left\langle E_{i}, \nabla_{E_{i}} X\right\rangle_{M}$ for a local orthonormal frame $\left\{E_{i}\right\}$ of $M$, we have

$$
\begin{aligned}
\Delta_{M} f & =\sum_{i=1}^{n}\left\langle E_{i}, \nabla_{E_{i}}\left(\operatorname{grad}_{M} f\right)\right\rangle_{M} \\
& =\sum\left[E_{i}\left\langle E_{i}, \operatorname{grad}_{M} f\right\rangle-\left\langle\nabla_{E_{i}} E_{i}, \operatorname{grad}_{\bar{M}} f\right\rangle\right] \\
& =\sum\left[E_{i}\left(E_{i} f\right)-\left\langle\nabla_{E_{i}} E_{i}, \operatorname{grad}_{M} f\right\rangle\right] \\
& =\sum\left[E_{i}\left(E_{i} f\right)-\left(\bar{V}_{E_{i}} E_{i}\right) f+\left\langle\alpha\left(E_{i}, E_{i}\right), \operatorname{grad}_{\bar{M}} f\right\rangle\right],
\end{aligned}
$$

where $\alpha(X, Y)=\bar{\nabla}_{X} Y-\nabla_{X} Y$ is the second fundamental form (cf. [25, pp. 11, 34]). Thus

$$
\Delta f=\sum \bar{\nabla}^{2} f\left(E_{i}, E_{i}\right)+n\left\langle H, \operatorname{grad}_{M} f\right\rangle,
$$

as required.

Proof of Theorem 3.1. Suppose that $M^{n}$ is contained in a ball of radius $R$ and center $x_{0} \in \bar{M}^{n+k}$. Set $f=\bar{\varrho}(x)^{2}$ where $\bar{\varrho}(x)=$ distance from $x$ to $x_{0}$ in $\bar{M}^{n+k}$. Recall that it follows from the hypotheses that $\bar{\varrho}^{2}$ is smooth (cf. [16], Chap. 1]). Using Lemma 3.2 we find

$$
\Delta_{M} f \geqq \operatorname{tr}_{M}\left[\bar{\nabla}^{2}\left(\tilde{\varrho}^{2}\right)(x)\right]-n H_{0} \cdot 2 \bar{\varrho}(x) \geqq 2 n-2 n H_{0} R .
$$

Here we have used the Hessian Comparison Theorem (cf. [30], and [13, Chap. 2]) according to which $\bar{\nabla}^{2}\left(\bar{\varrho}^{2}\right) \geqq 2\langle\cdot, \cdot\rangle_{\bar{M}}$ since $\bar{M}^{n+k}$ is simply-connected and of nonpositive curvature. Since $f$ is bounded on $M^{n}$ we must have $H_{0} R \geqq 1$, according to Theorem 2.3. The proof is complete.

Proof of Corollary 3.1.1. It suffices to show that $\gamma_{2}<\infty$ follows from the estimate on the scalar curvature in the presence of the condition $H_{0}<\infty$. For this we note that if $\left\{E_{i}\right\}$ is a local orthonormal frame for $M^{n}$ then contraction of the Gausscurvature equation gives

$$
S(x)=\sum_{i \neq j} \sec _{\bar{M}}\left(E_{i} \wedge E_{j}\right)+n^{2}\|H\|^{2}-\|\alpha\|^{2},
$$


where $\|\alpha\|^{2}$ denotes the square of the length of the second fundamental form $\alpha$. It follows that

$$
\|\alpha\|^{2} \leqq n^{2} H_{0}^{2}+C\left(1+\varrho(x)^{2}\right)+\bar{K}_{R},
$$

where $\bar{K}_{R}=n(n-1)$ times the maximum of the sectional curvatures of $\bar{M}$ on the ball of radius $R$ that contains $M^{n}$. It now follows from the Gauss curvature equation

$$
\sec _{M}(X \wedge Y)=\sec _{\bar{M}}(X \wedge Y)+\langle\alpha(X, X), \alpha(Y, Y)\rangle-\|\alpha(X, Y)\|^{2}
$$

for a two plane $X \wedge Y \subset T_{p} M \subset T_{P} \bar{M}$, that the sectional curvatures of $M^{n}$ satisfy $\left|\sec _{x}\right|$ $\leqq C^{\prime}\left(1+\varrho(x)^{2}\right)$ for some $C^{\prime}>0$. The desired conclusion now follows from Corollary 2.1.2.

Proof of Corollary 3.1.2. For a minimal immersion $H=0$ (i.e., $H_{0}=0$ ) and so the hypothesis concerning the scalar curvature leads to $\gamma_{2}<\infty$ as in the proof of Corollary 3.1.1. Of course, it actually suffices to assume that $\gamma_{2}<\infty$.

Remarks. (a) Under the more restrictive assumption that the scalar curvature is bounded below, the conclusions of Corollaries 3.1.1 and 3.1.2 were obtained by Aminov [1] for $n=2$ and $\bar{M}=\mathbb{R}^{N}$, and Hasanis and Koutroufiotis [14] for $n \geqq 2$ and $M=\mathbf{R}^{N}$, and Jorge and Xavier [20] for $n \geqq 2$ and $\bar{M}$ as above [19]. Weaker results, for $n \geqq 2$ and $M^{n}=\mathbb{R}^{N}$, were obtained earlier in [1] and [6]. See also [23] for a formulation of results slightly weaker than those given here.

(b) Similar estimates can be obtained for the exterior diameter of $M^{n}$ immersed with bounded mean curvature in a normal coordinate ball $B_{R}$ in any manifold $\bar{M}$. These involve the maximum curvature of $\bar{M}$ in $B_{R}$. For this one only needs lower estimates for $\bar{\nabla}^{2} \tilde{Q}^{2}$, as in the proof of Theorem 3.1, and these are readily available (in [13], for example). This is carried out under more restrictive hypotheses in [20] and [21].

c) The conclusions of Corollary 3.1.2 are not valid for aribtrary minimal immersions and some hypothesis such as $\gamma_{2}<\infty$ or $S(x) \geqq-C\left(1+\varrho(x)^{2}\right)$ is required, at least if the codimension is greater than one. In fact, there exist constructions of bounded two dimensional minimal surfaces in $\mathbb{R}^{n}, n \geqq 4$ due to Calabi (see [34, p. 690]) and Jones [18]. The question of Calabi (see [10]) and Chern [8] as to whether or not there exist bounded minimal submanifolds in $\mathbb{R}^{n}$ remains open in the case of codimension one, and the results of this paper are the most general known for arbitrary codimension.

d) For $M^{n}$ compact the result of Corollary 3.1.2 is due to O'Neill [28].

\section{Applications: The Heat Equation and Brownian Motion}

In this section we take up some connections between Brownian motion (or, equivalently, heat diffusion) on noncompact Riemannian manifolds and the previous results.

It is known that there exists a unique minimal heat kernel $p(x, y, t)$ for any noncompact complete Riemannian manifold $\left(M^{n}, d s^{2}\right)$ (cf. [3]; see [9] for a clear 
and detailed construction). Associated with this kernel, there is a unique Markov diffusion process $\left\{X_{t}\right\}$ called Brownian motion on $\left(M^{n}, d s^{2}\right)$, and it is known that $\left\{X_{t}\right\}$ is conservative (or nonexplosive), i.e., $\left\{X_{t}\right\}$ remains in $M^{n} C M^{*}(=$ the one point compactification of $M$ ) for all $0 \leqq t<\infty$, if and only if bounded solutions of the heat equation on $M^{n} \times[0, T]$ are uniquely determined by their initial data (cf. [3], or [24] for a more complete discussion).

We first note that

Theorem 4.1. Brownian motion is explosive on a complete bounded minimal submanifold of of $\mathbb{R}^{N}$.

Proof. The proof of Corollary 3.1.2 in conjunction with the proof of Theorem 2.3, shows that on a bounded minimal submanifold of $\mathbb{R}^{N}$ there exist two distinct bounded solutions of the heat equation $\left(\Delta-\frac{\partial}{\partial t}\right) u=0$ on $M x[0, T]$ with $u(x, 0) \equiv 0$, the function $\psi$ constructed in the proof of Theorem 2.3 and the function $u \equiv 0$.

On the other hand we have

Theorem 4.2. Brownian motion is non explosive on a minimal graph $x_{n+1}=f\left(x_{1}, \ldots, x_{n}\right)$ over $\mathbb{R}^{n}$, and even on any complete properly imbedded minimal submanifold $M^{n}$ of $\mathbb{R}^{N}$.

Remark. Recall that it is known that for $n \geqq 8$ there exist minimal graphs that are not planes [5], while for $n \leqq 7$ all minimal graphs are planes [29].

Proof. We will show that bounded solutions of the heat equation on $M x[0, T]$ are completely determined by their initial values. It can be shown that for area minimizing hypersurfaces of $\mathbb{R}^{N}$ this result follows from the very general results of [24]. It is our purpose here to give an elementary proof following standard methods, motivated by [9], which applies also to properly imbedded surfaces. To this end we consider the function $\phi=\sum_{i=1}^{N} x_{i}^{2}+(2 N+1) t$ restricted to $M^{n}$. It follows, as in Section three, that for the Laplacian of $M, \Delta$, we have $\left(\Delta-\frac{\partial}{\partial t}\right) \phi \leqq-1$. If $u(x, t)$ is a bounded solution of $\left(\Delta-\frac{\partial}{\partial t}\right) u=0$ on $M \times[0, T]$ then set

$$
\begin{aligned}
& N_{\mathrm{T}}=\sup _{M x\left[0^{\prime} r\right]}|u(x, t)| \\
& N_{0}=\sup _{M}|u(x, 0)|
\end{aligned}
$$

and

$$
v= \pm u-N_{0}-\frac{N_{T}}{R^{2}} \phi .
$$

Let $M_{R}$ be the portion of $M$ that intersects the $N$-ball $B_{R}(0)$. Then on $M_{R} \times[0, T]$ we have $\left(A-\frac{\partial}{\partial t}\right) v>0$, while $v<0$ on $\partial M_{R} \times[0, T] \cup M_{R} \times\{0\}$. It follows from the 
maximum principle that $v \leqq 0$ in $M_{R} x[0, T]$, i.e.,

$$
\pm u(x, t) \leqq N_{0}+\frac{N_{T}}{R^{2}} \phi(x, t) .
$$

Here we need to choose $R=R_{i}(\rightarrow \infty)$ such that $M_{R_{i}}$ is a compact manifold with boundary $\partial M_{R_{i}}=M \cap \partial B_{R_{i}}$. This is possible since $M$ is complete and properly imbedded in $\mathbb{R}^{N}$. Letting $R_{i} \rightarrow \infty$ while $(x, t)$ are fixed, we obtain

$$
|u(x, t)| \leqq \sup _{\Gamma}|u(x, 0)|
$$

which yields the desired conclusion.

\section{References}

1. Aminov, J.: The exterior dimaeter of an immersed Riemannian manifold. Math. USSR Sborn. 21, 449-454 (1973)

2. Andreotti, A., Vesentini: Carleman estimates for the Laplace-Beltrami operator on complex manifolds. Publ. Inst. Hautes Etudes Sci. 25, 81-130 (1965)

3. Azencott, R.: Behavior of diffusion semi-groups at infinity. Bull. Sci. Math. France 102, 193-240 (1974)

4. Bishop, R., Crittenden, R.: Geometry of manifolds. New York: Academic Press 1964

5. Bombieri, E., Giorgi, Ei Di, Giusti, E.: Minimal cones and the Bernstein problem. Invent. Math. 7, 243-268 (1969)

6. Burago, Ju.: On a theorem of J.A. Aminov (in Russian). In: Problems in global geometry. Zap. Naucn. Sem. Leningrad, Otdel. Math. Inst. Steklov 45, 53-55 (1974)

7. Cheng, S.Y., Yau, S.T.: Differential equations on Riemannian manifolds and their geometric applications. Comm. Pure Appl. Math. 28, 333-354 (1975)

8. Chern, S.S.: The geometry of $G$-structures. Bull. Am. Math. Soc. 72, 167-219 (1966)

9. Dodziuk, J.: Maximum principle for parabolic inequalities and the heat flow on open manifolds. Indiana Univ. Math. 32, 706-716 (1983)

10. Eells, J., Jr., Kobayashi, S.: Problems in differential geometry. In: Proceedings of U.S.-Japan Seminar on Differential Geometry, pp. 167-177. Kyoto, 1965

11. Friedman, A.: Partial differential equations of parabolic type. Englewood Cliffs:Prentice-Hall 1969

12. Gaffney, M.P.: The conservation property of the heat equation on Riemannian manifolds. Comm. Pure Appl. Math. 12, 1-11 (1959)

13. Greene, R., Wu, H.: Function theory on manifolds which possess a pole. In: Lecture Notes in Mathematics, No. 699. Berlin, Heidelberg, New York: Springer 1979

14. Hasanis, T., Koutroufiotis, D.: Immersions of bounded mean curvature. Arch. Math. 33, 170-171 (1979)

15. Hasminskii, R.Z.: Ergodic properties of recurrent diffusion processes. Theor. Appl. Probability Appl. 5, 179-196 (1960)

16. Helgason, S.: Differential geometry and symmetric spaces. New York: Academic Press 1962

17. Hiriart-Urruty, J.B.: A short proof of the variational principle for approximate solutions of a minimization problem. Am. Math. Monthly 90, 206-207 (1983)

18. Jones, P.: A complete bounded complex submanifolds of $C^{3}$. Proc. Am. Math. Soc. 76. 305-306 (1979)

19. Jorge, L.P.de M., Xavier, F.: On the existence of complete bounded minimal surfaces in $\mathbb{R}^{N}$. Bol. Soc. Bras. Mat. 10, 191-194 (1979)

20. Jorge, L.P.de M., Xavier, F.: An inequality between exterior diameter and mean curvature of bounded immersions. Math. Z. 178, 77-82 (1981) 
21. Jorge, L.P.de M., Koutroufiotis, D.: An estimate for the curvature of bounded submanifolds. Am. J. Math. 103, 711-725 (1981)

22. Karp, L.: Subharmonic functions on real and complex manifolds. Math. Z.179, 535-55 (1982)

23. Karp, L.: Subharmonic functions, harmonic mappings, and isometric immersions. In: Seminar on Differential Geometry, S. T. Yau, ed., pp. 133-142. Princeton: Princeton University Press 1982

24. Karp, L., Li, P.: The heat equation on complete Riemannian manifolds. To appear

25. Kobayashi, S., Nomizu, K.: Foundations of differential geometry, Vol. II. New York: Interscience 1969

26. Nash, J.: The embedding problem for Rieman manifolds. Ann. Math. 63, 20-63 (1956)

27. Omori, H.: Isometric immersions of Riemannian manifolds. J. Math. Soc. Japan 19, 205-214 (1967)

28. O'Neill, B.: Immersions of manifolds of nonpositive curvature. Proc. Amer. Math. Soc. 11, 132-134 (1960)

29. Simons, J.: Minimal varieties in Riemannian manifolds. Ann. Math. 88, 62-105 (1968)

30. Siu, Y.T., Yau, S.T.: Complete Kähler manifolds with nonpositive curvature of faster than quadratic decay. Ann. Math. 105, 225-264 (1977)

31. Yau, S.T.: Harmonic functions on complete Riemannian manifolds. Comm.Pure Appl. Math. 28, 201-228 (1975)

32. Yau, S.T.: Some function theoretic properties of complete Riemannian manifolds and their applications to geometry. Indiana Univ. Math. J. 25, 659-670 (1976)

33. Yau, S.T.: A general Schwarz lemma for Kähler manifolds. Am. J. Math. 100, 197-203 (1978)

34. Yau, S.T., ed.: Seminar on differential geometry. Princeton: Princeton University Press 1982

Received February 20, 1984

Note added in proof. A slight change in the function $\phi$ in the proof of Theorem 4.2 yields the same conclusion under the weaker hypothesis that $H$ is of polynomial growth in $|x|$. The details are left to the interested reader. 